\title{
Physical Bases of Work of Photo Receivers Based on Silicides
}

\author{
Elchin Ahmed Kerimov", Sevinj Nadir Musaeva ${ }^{2}$ \\ ${ }^{1}$ Department of Physics, Azerbaijan State Academy of Oil and Industry, Baku, Azerbaijan \\ ${ }^{2}$ Department of Electrical Engineering and Electrical Equipment, Azerbaijan Technical University, Baku, Azerbaijan
}

Email address:

E_Kerimov.fizik@mail.ru(E.A. Kerimov)

\section{To cite this article:}

Elchin Ahmed Kerimov, Sevinj Nadir Musaeva. Physical Bases of Work of Photo Receivers Based on Silicides. Science Journal of Chemistry. Vol. 8, No. 4, 2020, pp. 77-80. doi: 10.11648/j.sjc.20200804.11

Received: March 19, 2020; Accepted: April 24, 2020; Published: September 14, 2020

\begin{abstract}
The creation of high-quality high-speed semiconductor devices and integrated circuits requires the introduction of new materials into the technology for their manufacture. The most promising of them are silicides-silicon compounds with more electropositive elements. These compounds can be obtained as a result of a solid phase reaction at a temperature in the range of about one to half the melting point of this metal on an absolute scale. Silicides have a high conductivity of a metallic nature, high temperature stability, and surpass in these properties any heavily doped semiconductor layer. The use of polysilicon as a material for gates and connecting lines providing a layer resistance of $20 \mathrm{Ohms} / \square$ allowed us to reduce the minimum dimensions of the elements of devices to 25 microns. Methods were proposed for the formation of silicide films, as well as technological processes necessary for the manufacture of semiconductor devices and microcircuits with their application, which allowed us to start developing devices with a minimum element size of $1 \mu \mathrm{m}$ and to begin their industrial production. The stable and reliable characteristics of platinum-silicon silicide (PtSi-Si) contacts have led to the widespread use of silicides as materials for ohmic contacts, gates in metal-oxide-semiconductor (MIS)-transistors, materials for storing optical information, photodetectors operating in IR-spectral regions, etc. The Si-2p band has an asymmetric shape; the structure of valence states differs from metallic ones.
\end{abstract}

Keywords: Impurities, Phase, Silicon, Iridium Film, Platinum Film

\section{Introduction}

The conclusion is drawn about the strong interaction between $\mathrm{Si}$ and $\mathrm{Pt}$ up to coatings of 40 monolayers. When applying the method of single-layer metallization, uniformity decreases with increasing thickness of PtSi. The boundary homogeneity does not depend on the PtSi thickness in the case of "thin" PtSi-Si contacts obtained by multilayer metallization. In order to make contacts in this way, a Pt layer $150 \mathrm{~A}^{\circ}$ thick and several pairs of alternating silicon $\left(132 \mathrm{~A}^{\circ}\right)$ and platinum $\left(100 \mathrm{~A}^{\circ}\right)$ layers are sprayed onto a silicon substrate. The kinetics of the formation of platinum silicide in particular and the effect of impurities on the formation of PtSi were intensively studied by such methods as Auger spectroscopy, X-ray diffraction, Rutherford backscattering of $\mathrm{H}+\mathrm{ions}$, and scanning electron microscopy. It was found that in addition to the annealing temperature, impurities play an important role in the solid-phase growth of silicides, slowing down the reaction of their formation.

PtSi epitaxial films on $\mathrm{Si}<111>$ are more stable than nonepitaxial films on $<100>$ deposited at temperatures above $1000^{\circ} \mathrm{C}$. The influence of nitrogen atoms located in the surface Si layer and annealing temperature on the growth pattern of platinum silicide layers was studied [1-5]. Nitrogen atoms were introduced into the silicon substrate during its purification by bombardment with nitrogen ions. After such cleaning, platinum films with a thickness of $600 \mathrm{~A}^{\circ}$ were sprayed onto the surface of a Si substrate with an orientation of $\langle 100\rangle$ and a specific resistance of $8-12 \mathrm{Ohm} \cdot \mathrm{cm}$. The resulting structures were annealed in a stream of a gas mixture $\mathrm{N}_{2}-10 \% \mathrm{H}_{2}$ in the temperature range $500-6400^{\circ} \mathrm{C}$.

It was established that in the presence of nitrogen, PtSi grows in the form of "islands" of PtSi surrounded by the "sea". The diameter of the PtSi islands increases linearly with increasing annealing duration, and the growth rate is limited by the nucleation rate. Stresses in the $\mathrm{Pt}(\mathrm{PtSi}) / \mathrm{Si}$ structure 
determine the formation of microcracks in PtSi films and in the $\mathrm{Si}$ substrate. A model for the growth of PtSi from individual nucleation centers is proposed.

Oxygen is the main impurity that affects the formation of silicide. The oxygen content in the platinum films affected both the reaction rate and the phase growth sequence. For clean and dirty films, the diffusion coefficient differs by more than an order of magnitude. In addition, pure samples had a sharp interface, while for samples containing oxygen, it was rather uneven. The growth rate of silicides in the presence of a protective layer located on top of the platinum layer during annealing was higher compared to the growth of silicide with an unprotected platinum film on silicon. Such a layer protected the metal from the undesirable effect of small doses of oxygen and other contaminants contained in the working medium [6-9].

An understanding of the chemical nature of bulk compounds is especially important for studying the metalsilicon interphase with the aim of studying the nature of chemical bonds and charge redistributions for bulk metalsilicide transitions. Studies have shown that the strength of the interaction between metal and silicon increases in the following sequence:

\section{$\mathrm{Pt} 2 \mathrm{Si}>\mathrm{Pd} 2 \mathrm{Si}>\mathrm{Ni} 2 \mathrm{Si}$}

From the point of view of chemical resistance of PtSi determinations, the result of studies published in $[10,11]$ is of interest, in which a secondary oxidative annealing method is proposed to increase the chemical resistance of PtSi films to platinum dissolving reagents, and the effect of preliminary cleaning of the surface and the atmosphere in which annealing is performed is studied $\left(\mathrm{T}_{\text {anneal }}=400-5000 \mathrm{C}\right)$ on the quality of the films.

In light of this, the process of the formation of thin films of the Pt-Si system under controlled conditions was studied in [12-15], under which the kinetics and subsequent phase growth were found to depend on substrate heating during deposition. However, it was found that the presence of oxygen in the platinum film determines the reaction rate and phase growth, namely, the diffusion process of the formation of $\mathrm{PtSi}$ and $\mathrm{Pt}_{2} \mathrm{Si}$ and changes the phase sequence.

\section{The Theoretical Part}

When a metal and a semiconductor are brought into contact, depending on the relationship between the electron work function in the metal $\Phi_{M}$ and the $\chi+V_{P}$ semiconductor, electrons can transfer from metal to the semiconductor as a result of internal emission or vice versa. Figure 1 shows the band diagram of the contact between a metal silicide-p-type silicon for the case $\Phi_{\mathrm{M}}<\chi+\mathrm{V}_{\mathrm{P}}$. In this case, part of the electrons from the metal (silicide) passes into the semiconductor $(\mathrm{Si})$ until the thermodynamic equilibrium occurs and the Fermi levels in the metal and the semiconductor are aligned. Near the interface, a region depleted in charge carriers is formed in the semiconductor, a space charge region of uncompensated negative acceptor ions arises, the electric field of which prevents further emission of electrons from the metal into the semiconductor, and the energy zones of the semiconductor bend downward. If the thickness of the intermediate layer between the metal and the semiconductor is comparable with the interatomic distances, then the magnitude of the curvature-the height of the potential barrier-is equal to the contact potential difference

$$
\Psi_{M S}=\Phi_{S}-\Phi_{M}+\left(E_{f}-E_{V}\right)=\chi+E_{g}-\Phi_{M}
$$

where, the second term is the difference between the work function of the metal and the electron affinity of the semiconductor.

\section{Experimental Part}

Under the influence of IR radiation, two types of electronic transitions can occur in such a structure. If the energy of the incident photon is $h v \geq E_{g}$, then when it is absorbed in the semiconductor, electron-hole pairs are generated. In this case, as in the usual photodiode at the $\mathrm{p}-\mathrm{n}$ junction, carriers of different signs are separated by the transition field and a photo emf occurs.
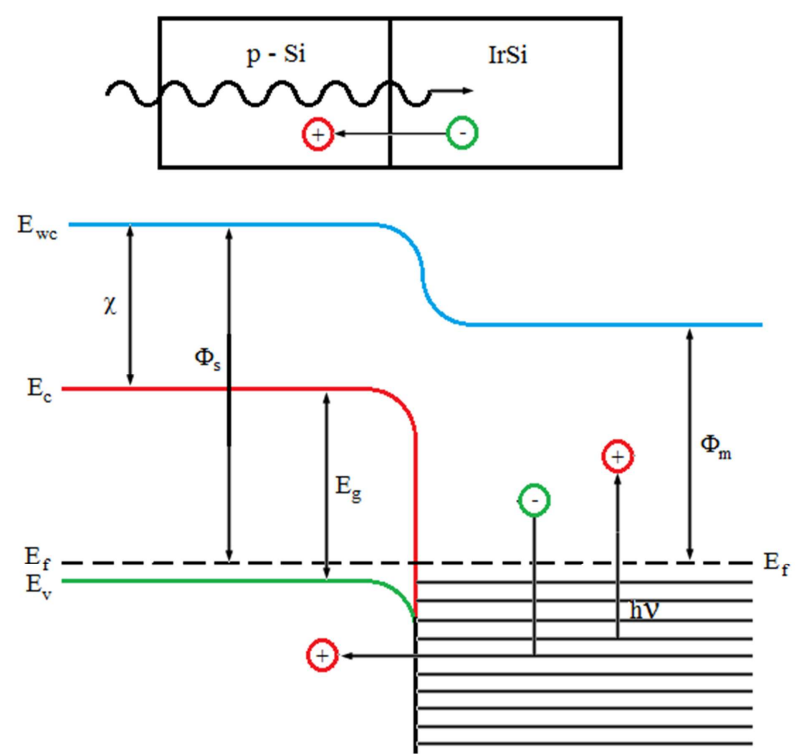

Figure 1. Energy diagram demonstrating the operation of the FDSB based on IrSi-p-Si.

Obviously, the long-wavelength boundary of such a process cannot be less than the semiconductor band gap, and from this point the FDSB (photodiode with a Schottky barrier) does not differ from $\mathrm{p}$-n photodiodes or intrinsic photoresistors. If $h v<\mathrm{E}_{\mathrm{g}}$, then the absorption of IR radiation in the metal film excites valence electrons in states above the Fermi level, leading to the appearance of holes, some of which have an energy greater than the barrier height. Then, either the hole is emitted from the metal into the semiconductor, or the electron passes from the semiconductor to the metal, filling the empty state.

To overcome the barrier during the transition from a metal 
to a semiconductor, the energy of an excited hole must be greater than the height of the barrier. The long-wavelength boundary of such a process can be changed by selecting the appropriate metal. Therefore, from the point of view of creating infrared radiation photodetectors on silicon, photoemissions from a metal to a semiconductor are of most interest.

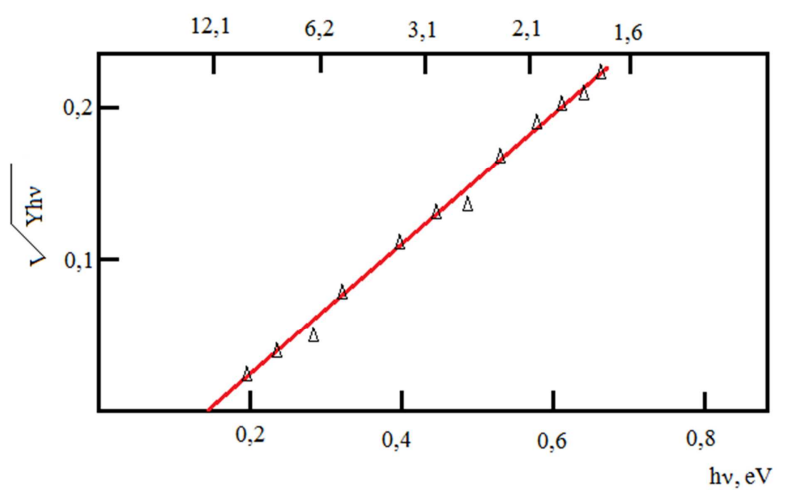

Figure 2. Dependence of the photoresponse on the energy of a quantum of light for a photodiode based on IrSi-p-Si.

Experiments and theoretical calculations show that the spectral dependence of the quantum yield $\mathrm{Y}$ of internal photoemission from a metal to a semiconductor can be described by the so-called refined Fowler formula $[1,2]$ :

$$
Y=C_{1} \frac{\left(h v-h v_{0}\right)^{2}}{h v}
$$

where, $\mathrm{C}_{1}$ is the coefficient of quantum photoemission, which is determined by the geometric, optical and physical properties of the Schottky contact. In figure 2 and figure 3 shows the dependence of the quantum yield observed for photodiodes based on IrSi-p-Si and PtSi-p-Si contacts.

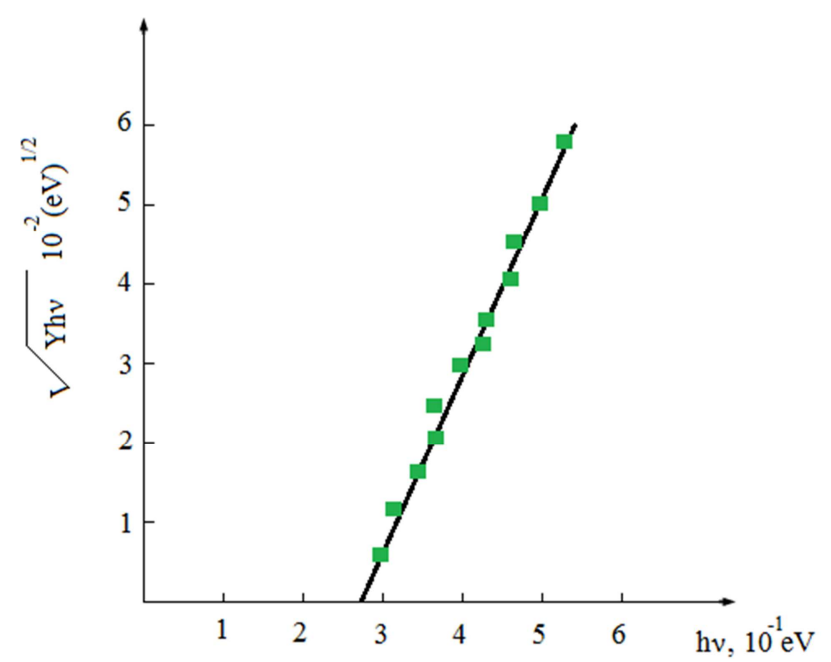

Figure 3. Dependence of the photoresponse on the quantum energy of light for a PtSi-p-Si based photodiode.

Table 1 shows the values of the corresponding longwavelength boundaries of the photoelectric effect $\lambda_{0}$ in the
FDSB based on the contact between metal silicide and silicon.

It should be emphasized that infrared photodetectors based on Schottky diodes in the form of spectral characteristics differ from ordinary intrinsic or impurity IR detectors. The quantum output of detectors based on an internal photoelectric effect (photoresistors or p-n photodiodes) does not depend on the quantum energy at an energy greater than the threshold value and is close to unity. For photodetectors on a SB (Schottky barrier), the quantum yield monotonically increases with the quantum energy at energies above the threshold.

Table 1. The values of the corresponding long-wavelength boundaries of the photoelectric effect.

\begin{tabular}{|c|c|c|}
\hline Silicide & $\Psi_{\mathrm{ms}}, \mathrm{eV}$ & $\lambda_{0}$, microns \\
\hline $\mathrm{TiSi}_{2}$ & 0,52 & 2,40 \\
\hline $\mathrm{TiSi}_{2}$ & 0,47 & 2,64 \\
\hline $\mathrm{NiSi}$ & 0,46 & 4,70 \\
\hline $\mathrm{Pt}_{2} \mathrm{Si}$ & 0,85 & 3,65 \\
\hline PtSi & 0,20 & 6,00 \\
\hline $\mathrm{Pd}_{2} \mathrm{Si}$ & 0,34 & 3,6 \\
\hline IrSi & 0,14 & 8,00 \\
\hline
\end{tabular}

\section{The Discussion of the Results}

The main processes occurring in the FBS are reduced to the absorption of photons in the silicide layer and the internal photoemission of carriers from the metal into the semiconductor. Therefore, the quantum yield and photosensitivity of these PDs (photodiodes) are determined mainly by electronic and optical processes in metal silicide, and not in a semiconductor. This means that the photosensitivity of the FDBS in the first approximation does not depend on such parameters of the semiconductor as the degree of doping and the level of compensation of impurities, as well as on the lifetime of minority current carriers. This eliminates the main causes of the uneven sensitivity in multielement matrices.

In addition, since the silicon substrate is not involved in the process of photogeneration of charge carriers, much less stringent requirements are imposed on it in terms of electrical parameters, and the same silicon that is used for the production of integrated circuits can be used for the fabrication of FSB. Compared to conventional photodetectors, FDSB have significantly lower detection ability at wavelengths close to the long-wavelength boundary.

Their dependence on the position of the long-wavelength photosensitivity boundary also differs: if, for ordinary photodetectors, a shift of the threshold wavelength $\lambda 0$ beyond the long-wavelength boundary of the transmission window does not at best reduce the detection ability, only if $\lambda_{0}$ is greater than the long-wavelength boundary of the corresponding transmission window. So, for a window of 3-5 $\mu \mathrm{m}$, the maximum value of the integrated detectability corresponds to $\lambda=7 \mu \mathrm{m}$.

This feature of the FDSB is due to a special type of 
spectral dependence of the quantum yield, when an increase in the long-wavelength boundary leads to an increase in quantum efficiency in a given wavelength range, which is not observed in conventional receivers. Thus, in the FDSB, the detection ability in the transparency windows of the atmosphere can be increased by using metals that provide a lower height of the potential barrier, but it is necessary to lower the cooling temperature of the sensitive element. The most promising photodiodes for detecting radiation in the region of 3-5 $\mu \mathrm{m}$ are photodiodes based on the PtSi-p-Si, $\mathrm{Pd}_{2} \mathrm{Si}$-p-Si, and IrSi-p-Si contacts.

\section{Basic Conclusions}

1. From theoretical calculations and experimental studies it can be concluded that the Y-spectral dependence of the quantum output of the internal photoemission from IrSi to the semiconductor is described by the refined Fowler formula.

2. It has been found that as the thickness of IrSi decreases, the absorption of infrared rays increases. Although this regularity is consistent with the considerations of classical optics (the Drude model), this dependence is the result of a quantum measurement effect.

\section{References}

[1] Yeo Yee-Chia, King Tsu-Jae, Hu Chenming // Metal-dielectric band alignment and its implications for metal gate complementary metal-oxide-semiconductor technology, Journal of applied physics, 2002, v. 92, No 12.

[2] Yudasaka M., Zhang M., Jabs C. et al. Effect of an organic polymer in purification and cutting of single-wall carbon nanotubes // Appl. Phys. A. 2000, v. 71, No 4, p. 449-451.

[3] Alemany L., Zhang L., Zeng L. et al. SolidState NMR Analysis of Fluorinated Single-Walled Carbon Nanotubes: Assessing the Extent of Fluorination // Chemistry of
Materials. 2007, v. 19, No 4, p. 735-744.

[4] Ando T. Theory of electronic states and transport in carbon nanotubes // Phys J. Sok. Japan. 2005, v. 74, p. 777.

[5] Baca A. Fabrication of GaAs devices. London, United Kingdom: The Institution of Electrical Engineers, 2005, p. 350 .

[6] Balan N., Gruzdev A., Nevsky A. / Books of abstracts, International conference "Micro-and nanoelectronics 2005", October 3rd-7th, 2005, Moscow, Zvenigorod, Russia. p. 1-11.

[7] Barrow J. Reducing Ground Bounce in DC-to-DC Converters-Some Grounding Essentials, Analog Dialogue. 2007, v. 41-2, p. 2-7.

[8] Belin T., Epron F. Characterization methods of carbon nanotubes: a review // Materials Science and Engineering B. 2005, v. 119, No 2, p. 105-118.

[9] Benton J., Kimerling L. Capacitance transient spectroscopy of trace contamination in silicon // J. Electrochem. Soc. 1982, v. 129, No 9, p. 2098-2102.

[10] Bhattacharya P., Properties of III-V quantum wells and superlattices, U.S.A., 1996.

[11] Brintlinger T., Chen Y., Durkop T., et al, Melingailis J. Rapid imaging of nanotubes on insulating substrates // Appl. Phys. Lett. 2002, v. 81, p. 2454-2456.

[12] Bulusheva L., Okotrub A., Guesel A. et al. Molecular Nanostructures. XVII Int. Winterschool / Euroconf. on Electronic Properties of Novel Materials / Eds Kuzmany H., Fink J., Mehring M., Roth S. AIP Conf. Proc. 685, 2003, p. 108.

[13] Chang C., Segmüller A. Huang H., et al. // Electrochem J. Soc. 1986, v. 133, № 6, p. 1256-1260.

[14] Chen Y., Fitzgerald J., Chadderton L., Of Metastable and Nanocrystalline Materials. 1999, v. 2, No 6, p. 375-380.

[15] Choi H., Ihm J., Louie S., et al, Phys. Rev. Lett. 84, 2000, p. 17-29. 